\title{
Estudio de rendimientos para las actividades estructura y mampostería para un proyecto de construcción en el campus de la UPB
}

\section{Labor productivity study about masonry and structure activities for a construction project at campus UPB}

\author{
Aldemar Remolina Millán ${ }^{1}$, Lina Maritza Polanco Sánchez ${ }^{2}$ \\ ${ }^{1}$ M. Sc. Universidad de los Andes. Profesor Asociado, Facultad de Ingeniería Civil, Universidad Pontificia Bolivariana, \\ Seccional Bucaramanga -Colombia. Grupo de Investigación en Gestión de Proyectos y Tecnologías de Construcción Sostenibles - GPTECs. \\ ${ }^{2}$ M.Sc (c) Ingeniería Civil de la Universidad Industrial de Santander. Bucaramanga, Colombia. \\ Email: aldemar.remolina@upb.edu.co
}

Recibido 20/05/14, Aceptado 10/06/2014

Citar como: A. Remolina, L. M. Polanco, "Labor productivity study about masonry and structure activities for a construction project at campus UPB", Prospect, Vol 12, N², 105-112, 2014.

\section{RESUMEN}

El presente artículo consolida los antecedentes, aspectos metodológicos y resultados de la investigación que permitió llevar a cabo el análisis de rendimientos de mano de obra correspondientes a la ejecución de algunas actividades de construcción tradicional, como son las estructuras de concreto y las obras de mampostería. El análisis fue realizado, tomando como base información real generada por la construcción del Edificio J en el campus de la Universidad Pontificia Bolivariana Seccional Bucaramanga. Dicho análisis se realizó con el fin de encontrar un estándar de rendimiento que permita la comparación de estas actividades en la realización de proyectos de características similares. El estudio evidenció las dificultades típicas que se presentan en lo relacionado con información disponible, herramientas y metodologías de toma de datos y análisis de resultados correspondiente a este tipo de mediciones; en consecuencia se generan propuestas metodológicas y de análisis con base en supuestos de carácter práctico que pueden ser tomados como referencia para futuras investigaciones. Los resultados de los rendimientos calculados se presentan finalmente en forma de índices de productividad, que servirán de base comparativa para futuros análisis.

Palabras clave: Rendimientos de Mano de Obra de Construcción; Rendimientos de Mampostería; Rendimientos de Estructura; Cuadrilla Equivalente.

\section{ABSTRACT}

This article consolidates the background, methodological aspects and results of the investigation that enabled to develop the analysis of labor productivity for execution of some typical building activities, such as concrete structures and masonry. The analysis was performed, based on actual information generated for construction of J Building at the Universidad Pontificia Bolivariana Sectional Bucaramanga campus. This analysis was performed in order to find a performance standard that allows comparison of these activities for similar projects. The study made evident the typical difficulties that arise, related to available information, tools, and methodologies to collect and analyze data and results for this type of labor; accordingly, some methodological and analysis proposals were generated based on practical assumptions which can be taken as reference for future researchs. Finally, the labor productivity results that were calculated are presented as productivity indexes which will serve as comparative basis to future studies.

Key words: Construction Labor Productivity; Masonry Productivity; Structure Productivity; Equivalent Crew. 


\section{INTRODUCCIÓN}

Uno de los aspectos que genera mayor incertidumbre al momento de proyectar el valor y la duración de una obra, sin lugar a dudas tiene que ver con la determinación de los rendimientos correspondientes a las diferentes actividades de construcción; de hecho los rendimientos que se utilizan para realizar estos cálculos, son tomados en el común de los casos de tablas que han sido elaboradas para unas condiciones de trabajo que no se especifican y con criterios que igualmente son una incógnita dado el nivel de generalización y simplificación con el que se dispone la información. Se ha llegado al extremo en muchos casos de utilizar estos rendimientos en forma errónea cuando han sido introducidos sin ningún tipo de evaluación o análisis en bases de datos y programas de computador que permiten elaborar presupuestos y programas de obra, dejando la responsabilidad del resultado final de la variable costo de los proyectos a factores de alta incertidumbre como los imprevistos y en el peor de los casos a la compensación de dichos errores a costa del sobre esfuerzo de la mano de obra que efectivamente realiza la actividad.

La investigación desarrollada llevó a cabo el análisis de rendimientos de mano de obra para algunas actividades de construcción tradicional de edificaciones, como son las estructuras de concreto y las obras de mampostería, basado en información real tomada de la construcción del Edificio J en el campus de la Universidad Pontificia Bolivariana Seccional Bucaramanga, con el objeto de establecer información con base en condiciones conocidas que pueda ser contrastada con fuentes estandarizadas como las bases de datos de rendimientos que se utilizan típicamente. Este trabajo permitió por otra parte definir un proceso metodológico para la toma de información directa en obra y deja planteada una alternativa para ajustar las duraciones reales por medio de cuadrillas equivalentes que faciliten la estandarización y ponderación de los resultados.

Aspectos relacionados con los antecedentes, el estado del arte y los conceptos teóricos sobre las bases de datos y los aspectos determinantes de los rendimientos de construcción, así como la descripción del proyecto objeto de estudio son presentados a continuación.

\section{BASE DE DATOS DE RENDIMIENTOS EN LA INDUSTRIA DE LA CONSTRUCCIÓN}

Una base de datos de rendimientos es un registro completo de datos sobre actividades típicas de construcción en donde se desagrega el consumo unitario de recursos para los insumos correspondientes a las categorías de materiales, equipo y mano de obra que se contabilizan de manera directa a la ejecución de la actividad. En Colombia las bases de datos que suministran información de rendimientos para actividades de construcción se empezaron a implementar por medio de pequeñas publicaciones esporádicas al inicio de la década del 80 siendo una de las pioneras en este campo la empresa Construdata la cual posteriormente se especializó en el desarrollo de software para el cálculo de presupuestos y el control de costos de construcción, llegando a consolidarse en el mercado como una de las más eficientes en este tipo de labores.

En la actualidad Construdata es un producto de la firma Legis S.A. y se ha consolidado como una revista especializada en el tema. Además de los datos de rendimientos y precios de insumos suministrados cada tres meses, se destacan los estudios de costos de construcción para cuatro de las principales ciudades del país como son: Bogotá, Cali, Medellín y Barranquilla. Tekhne Ltda es otra base de datos de naturaleza privada que se distribuye por medio de Internet y que también ha logrado una importante acogida en la industria de la construcción nacional. En ella se presenta un registro de precios de construcción cuya información se actualiza trimestralmente y proviene de diferentes fuentes como fabricantes, distribuidores y contratistas.

\section{ASPECTOS DETERMINANTES DE LOS RENDIMIENTOS}

El rendimiento de mano de obra está definido como el tiempo que emplea un obrero o una cuadrilla para ejecutar completamente una determinada actividad de construcción. Este se puede expresar en unidades de tiempo sobre unidades de la cantidad de obra ejecutada. El rendimiento se puede cuantificar por mediciones realizadas directamente en obra y está sujeto a las condiciones de trabajo de cada uno de los empleados [1]; por otra parte dependiendo de la finalidad de las mediciones realizadas algunos autores expresan el rendimiento de la mano de obra de construcción como la relación entre la cantidad de trabajo realizado por una cuadrilla sobre el tiempo en horas consumido para realizar dicho trabajo, a lo cual se le denomina $C L P$, sigla en inglés de Construction Labor Productivity [2].

Investigaciones doctorales realizadas en University of Dundee - UK, han cuantificado la influencia de los factores de la denominada edificabilidad -Buildability- en la productividad de los procesos de encofrado correspondiente a losas de concreto en edificaciones y recomiendan continuar estudios similares sobre cimentaciones, columnas, muros y vigas [3]. Farnad et al. [4] por su parte, realizaron una aproximación a la modelación de la productividad de la mano de obra de construcción basado en la dinámica de sistemas (SD - System Dynamics) y plantearon un modelo cualitativo que interrelacionada los 26 factores más signi- 
ficativos que afectan el rendimiento de la mano de obra, en una compleja estructura de bucles construida utilizando el análisis causa y efecto. En el entorno nacional, Botero [5], plantea como factores determinantes del rendimiento: la situación de la economía general y las condiciones de vinculación laboral propias de la región o el país, el clima de la zona y las variaciones propias de la época el año, el tipo de actividad constructiva a realizar, el equipamiento suministrado, las condiciones de supervisión técnica de los trabajos y particularmente el factor humano propio del trabajador que realiza la tarea.

\section{DESCRIPCIÓN DEL PROYECTO OBJETO DE ESTUDIO}

La edificación que fue objeto de estudio y como tal la fuente de los datos de rendimientos de las actividades de construcción que se utilizaron en la presente investigación, es el denominado edificio J, una construcción multipropósito de $7.749,71 \mathrm{~m}^{2}$ conformada por un sótano de $1.679 \mathrm{~m}^{2}$ destinado para funcionar como parqueadero y cuatro plantas o niveles de piso conformados por biblioteca, auditorio, oficinas, baterías de baños, aulas de clase, plazoletas y zonas de circulación. Los acabados generales de la edificación son en ladrillo a la vista. La edificación fue proyectada para funcionar como un sistema estructural tipo aporticado en concreto normalmente reforzado, conformado por vigas, columnas y placas de entrepiso aligeradas en una dirección. El sistema de cimentación contempló un sistema de zapatas rectangulares conectadas por vigas de amarre y un sistema de muros perimetrales de contención alrededor de la excavación. Considerando que el objeto de estudio lo constituyeron las actividades más representativas de los procesos constructivos denominados estructura y mampostería, a continuación se describen las características técnicas de cada una de las actividades seleccionadas para la toma de información.

Por tratarse de un edificio concebido para que su acabado principal fuera el ladrillo a la vista, la mampostería de este constituyó un ítem altamente representativo. Las principales actividades que conforman la mampostería del proyecto y sobre las cuales se realizaron mediciones de rendimiento fueron: Muros (M), Muros Divisorios (MD) y Muretes $(\mathrm{Mu})$, elaborados en Bloque H10 y Muro a la Vista (MV) y Enchape Columna (EC), elaborados en Ladrillo M29.

\section{METODOLOGÍA}

En lo correspondiente a la determinación de los tiempos y rendimientos asociados a las tareas de construcción, esta se puede llevar a cabo por medio de la aplicación de métodos tradicionales como el estudio de tiempos y movimientos o el estudio de rendimientos por promedios. El método del estudio de tiempos y movimientos es propio de la industria manufacturera y presupone unas condiciones altamente controladas, de todos los factores determinantes del rendimiento, lo cual en el caso particular de la industria de la construcción es de difícil aplicación, dadas las múltiples variables que se derivan de las prácticas tradicionales propias de la construcción in-situ. Por su parte el método de estudio de rendimientos promedios, se acomoda mejor a las condiciones sui-generis de la industria de la construcción, dado que de esta forma se conjugan y promedian las múltiples variables que se presentan típicamente en obra [1]. El método seleccionado para llevar a cabo las mediciones fue el de estudio de rendimientos promedios, este método implicó la recolección diaria de información de manera permanente y en diferentes circunstancias de obra durante el desarrollo de la construcción. La tabulación de los datos, requirió el diseño de formatos consolidados de fácil diligenciamiento que una vez procesados en hoja de cálculo permitieron obtener los promedios representativos de rendimiento para cada actividad.

En la toma de información se consideró el número de trabajadores que fue requerido para el desarrollo de la labor, especificando categoría entre oficial y ayudante, dado que el rendimiento efectivo de la actividad está directamente relacionado con la cantidad de oficiales y ayudantes que participan en la misma, pero al momento de definir los rendimientos base, se debió realizar la respectiva conversión a cuadrillas equivalentes de un oficial y un ayudante. De igual forma en la toma de datos se contempló el porcentaje de obra ejecutado al momento de registrar la información, el tiempo que demora el personal en realizar dicha actividad y los tiempos de inactividad o descuentos por diferentes razones. El análisis de los datos registrados para las diferentes actividades requirió diseñar y elaborar una matriz de cálculo en una hoja electrónica, que permitió por una parte registrar de manera detallada aspectos determinantes del rendimiento como las dimensiones de cada elemento, ubicación espacial, tipo de proceso desarrollado, fecha, hora y duración de la tarea, porcentaje de obra ejecutado, personal involucrado y aspectos del entorno que afectaron el rendimiento. Por otra parte la hoja de electrónica realizó los cálculos de volúmenes y superficies de obra ejecutada, cálculo de cuadrillas equivalentes y finalmente el cálculo del rendimiento promedio.

En el caso de las actividades correspondientes a la estructura, se determinaron rendimientos promedio en Horas por Metro Cúbico $\left(\mathrm{H} / \mathrm{m}^{3}\right)$ para las actividades Zapata, Columnas, Vigas de Amarre y Muros de Contención en los procesos de Refuerzo (R), Encofrado (E), Fundida (F) y Desencofrado (D) respectivamente. Para las actividades correspondientes a la Mampostería, se calcularon rendimientos promedio en Horas por Metro Cuadrado $\left(\mathrm{H} / \mathrm{m}^{2}\right)$ para las actividades Muro en ladrillo H10 conformada por 
las labores de Muro (M), Muro Divisorio (MD) y Murete $(\mathrm{Mu})$ por una parte y las actividades Muro en ladrillo M29 conformada por las labores Muro a la Vista (MV) y Enchape de Columnas (EC) por otra parte. La clasificación definida obedece esencialmente a la diferencia en las dimensiones del tipo de ladrillo utilizado lo cual determina en parte el rendimiento de la actividad. El rendimiento propuesto como rendimiento estándar, se calculó como el promedio ponderado de la relación entre el tiempo ajustado para la realización del trabajo respecto a la cantidad de obra ejecutada, correspondiente a cada una de las mediciones realizadas para los tipos de actividades y procesos definidos. Para el análisis de las actividades correspondientes al capítulo de estructura, se consideró que el rendimiento total de la actividad equivale a la suma aritmética de los rendimientos de los procesos de armado de refuerzo, encofrado, fundida y desencofrado.

\subsection{Toma de datos y procesamiento de la información}

Con el fin de llevar a cabo el procesamiento de la información tomada en obra y el correspondiente cálculo de rendimientos, se diseñaron cinco (5) matrices de datos en hojas electrónicas, correspondientes con cada una las actividades analizadas: Zapatas, Columnas, Vigas, Muros de Contención, y Mampostería. Cada matriz permitió registrar información detallada del tipo de elemento, su ubicación en planta y altura respecto a las convenciones de los planos, datos sobre la geometría y dimensiones del elemento y datos sobre las mediciones de tiempo para la realización de la actividad y la configuración de la mano de obra.

En lo referente a la ejecución de la tarea, se especificó el tipo de proceso ejecutado siempre y cuando este fuera parte sustancial de la actividad. En cada caso se registró el día y la hora de inicio y finalización de la observación y el porcentaje de avance de la actividad correspondiente a dicho lapso de tiempo, los descuentos de tiempo debido a suspensiones producto de variables no imputables a la actividad y la composición de la cuadrilla de trabajo expresada en número de ayudantes y oficiales que participaron directamente en la fabricación del elemento. Con esta información se calculó la Cuadrilla equivalente (Ce), las duraciones bruta y neta de la actividad, la proyección de la actividad para su terminación hasta el $100 \%$ y el rendimiento de la mano de obra expresado en Hora por Metro Cúbico Cuadrilla.

\subsection{Estimación de rendimientos y cálculo de la Cuadri- lla Equivalente (Ce)}

En la práctica, el trabajo requerido para la construcción de un elemento determinado de la edificación, es ejecutado por una cuadrilla de hombres cuyo número y nivel de competencia es determinado por la complejidad y el tama- ño de la actividad, de manera que mientras para desarrollar una actividad como levantar un muro en bloque H10, normalmente basta con un oficial experto en el tema y un ayudante para llevar a cabo tareas no calificadas como mezclar y transportar material, para la fundida de un placa de entrepiso, se puede llegar a requerir más de una docena de hombres entre oficiales y ayudantes que desarrollen distintas labores en simultánea (vaciar el concreto, esparcirlo uniformemente con pala, operar el vibrador, allanar la superficie, etc.). En consecuencia a la hora de determinar los rendimientos, el número de personas involucradas en la tarea afecta de manera directa el tiempo que demora en ejecutarse la actividad, de modo que a mayor personal involucrado es de esperarse una menor duración de la tarea y en consecuencia un mayor rendimiento.

Investigadores de la Universidad Nacional de San Juan en Argentina, han profundizado en el análisis de tiempos de duración de tareas de construcción en función de la conformación de la cantidad de oficiales y ayudantes que integran las cuadrillas usuales y proponen una metodología para configurar una cuadrilla óptima que genere la menor pérdida de tiempo y por consiguiente optimice el rendimiento. El modelo propuesto establece por una parte que el contenido total del trabajo de la cuadrilla ideal se obtiene como la suma del contenido de trabajo del oficial y el contenido de trabajo del ayudante; y en segundo lugar que la relación entre los contenidos de trabajo de oficial y ayudante, equivale a la relación entre la cantidad de oficiales y ayudantes integrantes de la cuadrilla ideal, como se indica en la ecuación (1) [6].

$$
\gamma_{i}=\frac{N^{\circ}{ }_{o f}}{N^{\circ}{ }_{a y}}
$$

De acuerdo a lo anterior, se puede establecer que cuando se desea estandarizar un rendimiento con fines de comparación o para establecer una base de datos de referencia, se hace necesario definir el rendimiento respecto a una cuadrilla base conformada por un solo oficial y un solo ayudante, de manera que el tiempo utilizado para realizar la actividad con cualquier cuadrilla de hombres sea equivalente al tiempo utilizado por un número mayor de cuadrillas base ejecutando simultáneamente la misma actividad. Al número de cuadrillas base requerida para ejecutar la misma actividad en el mismo tiempo que la llevó a cabo la cuadrilla real, se le denomina Cuadrilla Equivalente (Ce). En la práctica las cuadrillas reales no se conforman de igual número de oficiales y ayudantes de modo que permita conformar parejas exactas, por lo general existe una mayor proporción de ayudantes sobre la cantidad de oficiales, lo cual es comprensible toda vez que entre más compleja la actividad será mayor la necesidad de apoyo en labores no calificadas; esta situación define el protagonismo del oficial en la determinación del rendimiento de la 
actividad, lo cual quiere decir que sin importar la cantidad de ayudantes que lo apoyen, es el oficial el recurso que define la duración de la actividad y como tal el número de oficiales de la cuadrilla real determina el número de cuadrillas equivalentes, tal como se muestra en la ecuación (2).

$$
C_{e}=N^{\circ}{ }_{o f}
$$

A manera de ilustración, si una cuadrilla de mampostería conformada por cuatro oficiales y cuatro ayudantes construyen 20 metros cuadrados de muro en 10 horas, el rendimiento de dicha cuadrilla será $0.5 \mathrm{~h} / \mathrm{m}^{2}$, no obstante si se separa a dicha cuadrilla en cuatro frentes de trabajo cada uno con una cuadrilla base conformada por 1 oficial y 1 ayudante, es de esperarse que cada cuadrilla base desarrolle 5 metros cuadrados en las mismas 10 horas para un rendimiento de la cuadrilla base de $2 \mathrm{~h} / \mathrm{m}^{2}$, lo cual representa una disminución del rendimiento o una pérdida en la productividad. Lo anterior permite inferir que el rendimiento de la cuadrilla base se puede obtener de multiplicar el rendimiento de la cuadrilla real por el factor que se ha denominado cuadrilla equivalente (Ce), en otras palabras y continuando con el ejemplo, como una cuadrilla real conformada por 4 oficiales y 4 ayudantes equivale a cuatro cuadrillas base conformadas por 1 oficial y 1 ayudante, el tiempo gastado por cada cuadrilla base para realizar la misma cantidad de obra será 4 veces el tiempo de la cuadrilla real y como tal el rendimiento de la cuadrilla base ( $\mathrm{Rcb}$ ) de obtendrá de multiplicar por 4 el rendimiento de la cuadrilla real (Rcr), tal como lo ilustra la ecuación (3).

$$
R c b=R c r * C e
$$

Por otra parte, la cantidad de ayudantes que apoyan la labor del oficial, afectan el rendimiento de manera marginal, en la medida que aportan un porcentaje de eficiencia en proporción al número de ayudantes asignados a la tarea, pero hasta un límite que no podrá ser mejorado por más ayudantes que utilice. Ovararin et al. [7], analizaron 16 factores de campo que afectan significativamente la productividad en las tareas de mampostería correspondientes a la construcción de edificios y encontraron que se presenta una pérdida de productividad entre el $10 \%$ y el $36 \%$ asociado a cambios en la conformación de la cuadrilla de trabajo que variaron entre menores y severos. De igual forma, estudios realizados por Martínez et al. [8], en la Universidad Católica de Chile, han demostrado que el trabajo no productivo representa un $30 \%$ del tiempo total de trabajo en las obras construidas en chile; este problema se debe fundamentalmente a la ineficiencia en el manejo de la mano de obra producto de cuatro aspectos principalmente: Viajes Excesivos, Esperas y Detenciones, Trabajos Inefectivos y Trabajos Rehechos. Por su parte Polanco [9] estimó que por cada ayudante adicional que apoye el trabajo de la cuadrilla base, se puede obtener un aumento de eficiencia o reducción del tiempo de la tarea del orden del $10 \%$, considerando un límite de 3 ayudantes adicionales como máximo, dado que después de esta cantidad lo que se presenta es un exceso de recurso cuyo impacto sobre el rendimiento será contraproducente. De acuerdo a lo anterior, la productividad de la cuadrilla base se debe ajustar por un factor de eficiencia (Fa) producto de la cantidad de ayudantes adicionales a la cuadrilla base que se dispongan para desarrollar la tarea. Dicho factor puede ser calculado con la aplicación de la ecuación (4), en donde ( $\mathrm{Fa}$ ) es el factor de ajuste de la productividad, ( $\left.N^{\circ} a y\right)$ es el número de ayudantes de la cuadrilla real y $\left(N^{\circ} \circ f\right)$ es el número de oficiales de la cuadrilla real. En consecuencia el cálculo del rendimiento ajustado de la cuadrilla base se realiza a partir de la ecuación (5).

$$
\begin{gathered}
F a=1-\left[0.1 *\left(\frac{N_{a y}^{\circ}}{N_{o f}^{\circ}}-1\right)\right] \\
R c b=R c r * C e * F a
\end{gathered}
$$

El análisis anterior encuentra respaldo en trabajos como el realizado por Mejía et al. [10], quienes plantean que la producción de una cuadrilla tipo está definida sustancialmente por tres aspectos: la composición de la cuadrilla, el proceso a desarrollar y las condiciones del entorno. De los aspectos identificados, los dos primeros determinan la eficiencia básica del trabajo a realizar y los aspectos relacionados con el entorno constituyen los factores de afectación. Al momento de Valorar el rendimiento, se debe considerar el factor de afectación que modificará la eficiencia tipo castigando o contribuyendo a mejorar la eficiencia. En su trabajo denominado Rendimientos y Consumos de Mano de Obra, Cano et al. [11], proponen como factor de afectación correspondiente a las habilidades del trabajador, un rango de porcentajes de eficiencia entre 60 y $75 \%$. Por su parte Mejía et al. [10], emplean el 70\% como valor para la eficiencia tipo.

\section{DISCUSIÓN Y ANÁLISIS DE RESULTADOS}

La estimación de rendimientos para cada una de las actividades seleccionadas, fue el resultado de promediar los rendimientos de la cuadrilla base calculada para cada una de las observaciones realizadas en cada proceso de fabricación de acuerdo al tipo de elemento. A continuación se muestran los resultados obtenidos para cada una de las actividades seleccionadas y los correspondientes procesos observados, en donde se indica para cada actividad y proceso la cantidad de mediciones realizadas, el volumen o superficie total medido y el respectivo promedio de acuerdo a la cantidad de mediciones y el rendimiento promedio para cada proceso expresado en horas por metro cúbico para las actividades de estructura y en horas por metro cuadrado para las actividades de mampostería. 
En lo correspondiente a la actividad zapatas, se llevaron a cabo 7 observaciones distintas de las cuales 2 corresponden al proceso de armado de refuerzo, 2 al proceso de encofrado y 3 al proceso de desencofrado. En la tabla 1 se puede observar que en promedio los rendimientos para estos tres procesos fueron de $0.03,2.7$ y 0.71 horas por metro cúbico respectivamente. En suma estos tres procesos totalizan $3.44 \mathrm{~h} / \mathrm{m}^{3}$ sin contar con el tiempo de fundida de la zapata. Construdata [12] registra un rendimiento de $8.27 \mathrm{~h} / \mathrm{m}^{3}$ para la misma actividad el cual podemos estimar que está bastante alto considerando que la fundida de no puede superar los $2.5 \mathrm{~h} / \mathrm{m}^{3}$ según los cálculos de rendimiento obtenido para este mismo proceso en actividades de columnas y vigas de concreto.

Tabla 1. Rendimientos Promedio para Zapatas.

Table 1. Average Performance for Footing.

\begin{tabular}{|c|c|c|c|}
\hline $\begin{array}{c}\text { Proceso/ } \\
\text { Actividad }\end{array}$ & Mediciones & $\begin{array}{c}\text { Volumen } \\
\mathbf{m}^{\mathbf{3}}\end{array}$ & $\begin{array}{c}\text { Rendimiento } \\
\mathbf{h} / \mathbf{m}^{3}\end{array}$ \\
\hline Desencofrado & $\mathbf{3}$ & $\mathbf{9 . 8 6}$ & $\mathbf{0 . 7 1}$ \\
\hline Zapata Z5 & 2 & 5.81 & 0.62 \\
\hline Zapata Z6 & 1 & 4.06 & 0.88 \\
\hline Encofrado & $\mathbf{2}$ & $\mathbf{1 . 7 3}$ & $\mathbf{2 . 7 0}$ \\
\hline Zapata Z1 & 2 & 1.73 & 2.70 \\
\hline Refuerzo & $\mathbf{2}$ & $\mathbf{6 . 9 6}$ & $\mathbf{0 . 0 3}$ \\
\hline Zapata Z5 & 1 & 2.90 & 0.02 \\
\hline Zapata Z6 & 1 & 4.06 & 0.04 \\
\hline Total general & $\mathbf{7}$ & $\mathbf{1 8 . 5 5}$ & $\mathbf{1 . 0 9}$ \\
\hline
\end{tabular}

En el caso de las columnas de concreto, se realizaron en total 37 observaciones entre columnas de sección rectangular y sección circular con diferentes dimensiones distribuidas de la siguiente forma: 14 mediciones corresponden al proceso de armado de refuerzo, 14 mediciones al proceso de encofrado, 1 medición al proceso de fundida, y 8 mediciones al proceso de desencofrado. Los resultado de rendimiento obtenidos son los siguientes: El armado de refuerzo toma en promedio 3.47 horas por cada metro cúbico de columna, el encofrado toma 2.54 horas por metro cúbico, la fundida toma 2.18 horas por metro cúbico y el desencofrado toma 1.03 horas por metro cúbico. Si se suman todos estos rendimientos en forma aritmética tendremos un total de 8.95 horas por metro cúbico de columna; Construdata [12] registra un rendimiento de $10.29 \mathrm{~h} /$ $\mathrm{m}^{3}$ para la misma actividad el cual podemos observar que es un $15 \%$ mayor al rendimiento medido en esta investigación. En la tabla 2 se pueden detallar los rendimientos obtenidos para cada tipo de columna observada.
Tabla 2. Rendimientos Promedio para Columnas.

Table 2. Average Performace for Columns.

\begin{tabular}{|c|c|c|c|}
\hline $\begin{array}{c}\text { Proceso/ } \\
\text { Actividad }\end{array}$ & Mediciones & $\begin{array}{c}\text { Volumen } \\
\mathbf{~ m}^{\mathbf{3}}\end{array}$ & $\begin{array}{c}\text { Rendimiento } \\
\mathbf{h} / \mathbf{m}^{\mathbf{3}}\end{array}$ \\
\hline Desencofrado & $\mathbf{8}$ & $\mathbf{8 . 2 5}$ & $\mathbf{1 . 0 3}$ \\
\hline C. Rectangular & 8 & 8.25 & 1.03 \\
\hline Encofrado & $\mathbf{1 4}$ & $\mathbf{1 2 . 3 6}$ & $\mathbf{2 . 5 4}$ \\
\hline C. Circular & 3 & 1.44 & 3.76 \\
\hline C. Rectangular & 11 & 10.93 & 2.21 \\
\hline Fundida & $\mathbf{1}$ & $\mathbf{0 . 9 6}$ & $\mathbf{2 . 1 8}$ \\
\hline C. Rectangular & 1 & 0.96 & 2.18 \\
\hline Refuerzo & $\mathbf{1 4}$ & $\mathbf{1 3 . 9 0}$ & $\mathbf{3 . 4 7}$ \\
\hline C. Rectangular & 14 & 13.90 & 3.47 \\
\hline Total & $\mathbf{3 7}$ & $\mathbf{3 5 . 4 8}$ & $\mathbf{2 . 5 5}$ \\
\hline
\end{tabular}

En lo referente a las vigas de concreto se llevaron a cabo 27 observaciones entre vigas de diferente sección distribuidas de la siguiente forma: 17 mediciones corresponden al armado de refuerzo que dieron como promedio 3.93 horas por metro cúbico de viga, 1 medición correspondiente al encofrado de la viga que dio como resultado un rendimiento de $1.93 \mathrm{~h} / \mathrm{m}^{3}, 4$ mediciones correspondientes a la fundida de la viga que dieron como resultado un rendimiento promedio de $2.45 \mathrm{~h} / \mathrm{m}^{3}$ y 5 mediciones correspondientes al desencofrado de la viga que promediaron 1.41 $\mathrm{h} / \mathrm{m}^{3}$ de viga. En total la suma de los rendimientos promedio de estos cuatro procesos alcanza un valor de $9.72 \mathrm{~h} / \mathrm{m}^{3}$ de viga, el cual se encuentra un $29 \%$ por encima de las 6.89 $\mathrm{h} / \mathrm{m}^{3}$ que suministra Construdata [12] para la misma actividad. En la tabla 3 se pueden observar los rendimientos promedio que sirvieron de base para el análisis.

Tabla 3. Rendimientos Promedio para Vigas.

Table 3. Average Performance for Beams.

\begin{tabular}{|c|c|c|c|}
\hline $\begin{array}{c}\text { Proceso/ } \\
\text { Actividad }\end{array}$ & Mediciones & $\begin{array}{c}\text { Volumen } \\
\mathbf{m}^{\mathbf{3}}\end{array}$ & $\begin{array}{c}\text { Rendimiento } \\
\mathbf{h} / \mathbf{m}^{3}\end{array}$ \\
\hline Desencofrado & $\mathbf{5}$ & $\mathbf{3 . 1 4}$ & $\mathbf{1 . 4 1}$ \\
\hline Viga $0.4^{*} 0.4$ & 5 & 3.14 & 1.41 \\
\hline Encofrado & $\mathbf{1}$ & $\mathbf{0 . 7 7}$ & $\mathbf{1 . 9 3}$ \\
\hline Viga $0.4^{*} 0.4$ & 1 & 0.77 & 1.93 \\
\hline Fundida & $\mathbf{4}$ & $\mathbf{2 . 9 5}$ & $\mathbf{2 . 4 5}$ \\
\hline Viga $0.4^{*} 0.4 / 0.6$ & 4 & 2.95 & 2.45 \\
\hline Refuerzo & $\mathbf{1 7}$ & $\mathbf{1 0 . 8 8}$ & $\mathbf{3 . 9 3}$ \\
\hline Viga $0.4^{*} 0.4 / 0.6$ & 17 & 10.88 & 3.93 \\
\hline Total & $\mathbf{2 7}$ & $\mathbf{1 7 . 7 4}$ & 3.17 \\
\hline
\end{tabular}

La cuantificación de rendimientos para la actividad muros de contención en concreto reforzado, se realizó con base en 
un total de 15 observaciones para los procesos de refuerzo, encofrado, fundida y desencofrado, correspondientes a secciones de muro con alturas de 3.21 metros y 2.76 metros. El rendimiento promedio obtenido con base en 3 observaciones del proceso armado de refuerzo de muro fue de $0.42 \mathrm{~h} / \mathrm{m}^{3}$. El encofrado de los muros fue llevado a cabo con un rendimiento promedio de $1.36 \mathrm{~h} / \mathrm{m}^{3}$ producto de 2 observaciones.

Para la actividad fundida de muros de contención, se realizaron 8 observaciones que dieron como resultado un rendimiento promedio de $0.52 \mathrm{~h} / \mathrm{m}^{3}$. Por último la actividad desencofrado se realizó con un rendimiento promedio de $0.71 \mathrm{~h} / \mathrm{m}^{3}$ producto de 2 observaciones. La suma de los rendimientos promedio para los 4 procesos que conforman la actividad muros de contención en concreto da como resultado un rendimiento de $3.01 \mathrm{~h} / \mathrm{m}^{3}$; valor que comparado con el que plantea Construdata [12] para la misma actividad, tan solo alcanza a ser el 30\% de dicho rendimiento. Ver tabla 4.

Tabla 4. Rendimientos Promedio para Muros de Contención. Table 4. Average Performance for Retaining Wall.

\begin{tabular}{|c|c|c|c|}
\hline $\begin{array}{c}\text { Proceso/ } \\
\text { Actividad }\end{array}$ & Mediciones & $\begin{array}{c}\text { Volumen } \\
\mathbf{~}^{\mathbf{3}}\end{array}$ & $\begin{array}{c}\text { Rendimiento } \\
\mathbf{h} / \mathbf{m}^{\mathbf{3}}\end{array}$ \\
\hline Desencofrado & $\mathbf{2}$ & $\mathbf{7 . 7 0}$ & $\mathbf{0 . 7 1}$ \\
\hline Muro h=3.21 & 2 & 7.70 & 0.71 \\
\hline Encofrado & $\mathbf{2}$ & $\mathbf{8 . 6 7}$ & $\mathbf{1 . 3 6}$ \\
\hline Muro h=3.21 & 2 & 8.67 & 1.36 \\
\hline Fundida & $\mathbf{8}$ & $\mathbf{3 0 . 5 3}$ & $\mathbf{0 . 5 2}$ \\
\hline Muro h=2.76 & 1 & 3.31 & 1.08 \\
\hline Muro h=3.21 & 7 & 27.22 & 0.44 \\
\hline Refuerzo & $\mathbf{3}$ & $\mathbf{1 0 . 9 3}$ & $\mathbf{0 . 4 2}$ \\
\hline Muro h=2.76 & 2 & 6.62 & 0.44 \\
\hline Muro h=3.21 & 1 & 4.30 & 0.40 \\
\hline Total & $\mathbf{1 5}$ & $\mathbf{5 7 . 8 3}$ & $\mathbf{0 . 6 4}$ \\
\hline
\end{tabular}

En lo correspondiente a la medición de rendimientos para las actividades de mampostería, se llevaron a cabo 16 mediciones para los procesos de levantar muros, muros divisorios y muretes en bloque $\mathrm{H} 10$ obteniendo rendimientos muy similares en cada uno de los proceso, los cuales fueron realizados con un promedio de $0.33 \mathrm{~h} / \mathrm{m}^{2}$. Este valor está por debajo de los $0.72 \mathrm{~h} / \mathrm{m}^{2}$ que establece Construdata [12] para este mismo tipo de actividad. Por su parte, el rendimiento de la actividad ladrillo M29 fue analizado específicamente para dos procesos que guardan estrecha relación, el levantamiento de muros con ladrillo a la vista y el enchape de columnas con el mismo tipo de ladrillo a la vista. En el primer caso el rendimiento obtenido fue el promedio de 15 observaciones y su valor alcanzó $0.64 \mathrm{~h} / \mathrm{m}^{2}$. En el caso del enchape de columnas el rendimiento promedio para 12 observaciones realizadas fue de $0.43 \mathrm{~h} / \mathrm{m}^{2}$. En con- traste se citan los estudios realizados por Refaat et al. [13], quienes evaluaron la productividad de la mano de obra en actividades de mampostería a partir de una muestra de once proyectos de construcción en Egipto entre edificios comerciales y residenciales; en el citado estudio, la recolección de datos se llevó acabo diariamente por un periodo de cuatro meses y permitió determinar un rendimiento medio de $0.608 \mathrm{~h} / \mathrm{m}^{2}$ de mampostería, calculado con base en datos de productividad acumulada obtenidos en función del número de trabajadores que conformaba la cuadrilla, las horas de trabajo requeridas y la cantidad de obra construida en dicho tiempo. En general el promedio de rendimiento para la actividad Ladrillo M29 fue de $0.55 \mathrm{~h} / \mathrm{m}^{2}$. Este valor está por debajo de los $1.06 \mathrm{~h} / \mathrm{m}^{2}$ que establece Construdata [12] para este mismo tipo de actividad. Ver tabla 5.

Tabla 5. Rendimientos Promedio para Mampostería. Table 5. Average Performance for Masonry.

\begin{tabular}{|c|c|c|c|}
\hline $\begin{array}{c}\text { Proceso/ } \\
\text { Actividad }\end{array}$ & Mediciones & Área $\mathbf{~ m}^{\mathbf{2}}$ & $\begin{array}{c}\text { Rendimiento } \\
\mathbf{h} / \mathbf{m}^{\mathbf{2}}\end{array}$ \\
\hline Bloque H-10 & $\mathbf{1 6}$ & $\mathbf{1 4 8 . 2 4}$ & $\mathbf{0 . 3 3}$ \\
\hline Muro & 8 & 78.73 & 0.29 \\
\hline Muro Divisorio & 4 & 45.73 & 0.36 \\
\hline Murete & 4 & 23.78 & 0.38 \\
\hline Ladrillo M29 & $\mathbf{2 7}$ & $\mathbf{3 1 2 . 8 6}$ & $\mathbf{0 . 5 5}$ \\
\hline Enchape Columna & 12 & 125.57 & 0.43 \\
\hline Muro a la Vista & 15 & 187.29 & 0.64 \\
\hline Total & $\mathbf{4 3}$ & $\mathbf{4 6 1 . 1 0}$ & 0.47 \\
\hline
\end{tabular}

En la tabla 6, se consolidan en primer lugar los resultados de los rendimientos promedio obtenidos para las actividades Zapatas, Columnas, Vigas y Muros de Contención, en los procesos de Refuerzo (R), Encofrado (E), Fundida (F) y Desencofrado (D) calculado en horas por metro cúbico de concreto; dichos resultados se totalizan y se comparan con los rendimientos que para las mismas actividades han sido propuestos por Construdata [12], calculando el porcentaje de diferencia. De manera similar se proceden en el caso de las actividades de Mampostería en Bloque H10 y Ladrillo M29.

Tabla 6. Comparativo de Rendimientos.

Tabla 6. Comparison of Performances.

\begin{tabular}{|l|c|c|c|c|c|c|c|}
\hline ACTIVIDAD & R & E & F & D & TOTAL & Construdata & \% Dif \\
\hline Zapatas & 0.03 & 2.70 & & 0.71 & 3.44 & 8.27 & $-58 \%$ \\
\hline Columnas & 3.47 & 2.54 & 2.18 & 1.03 & $\mathbf{9 . 2 2}$ & 10.29 & $-10 \%$ \\
\hline Vigas & 3.93 & 1.93 & 2.45 & 1.41 & $\mathbf{9 . 7 1}$ & 6.89 & $41 \%$ \\
\hline $\begin{array}{l}\text { Muros de } \\
\text { Contención }\end{array}$ & 0.42 & 1.36 & 0.52 & 0.71 & $\mathbf{3 . 0 2}$ & 10.68 & $-72 \%$ \\
\hline Mampostería en Bloque H10 & & $\mathbf{0 . 3 3}$ & 0.72 & $-54 \%$ \\
\hline Mampostería en Ladrillo M29 & $\mathbf{0 . 5 5}$ & 1.06 & $-49 \%$ \\
\hline
\end{tabular}




\section{CONCLUSIONES}

En primer lugar al observar la actividad de armado de refuerzo (tabla 6), se puede evidenciar que los resultados de rendimiento obtenidos reflejan el grado de complejidad de la actividad. Es claro que el nivel de complejidad más bajo corresponde al armado de refuerzo de las zapatas en donde normalmente se elaboran parrillas rectangulares de acero que sin mayor dificultad se ubican sobre el terreno; le siguen en mayor nivel de complejidad las armadas de refuerzo de los muros de contención, las columnas y finalmente las vigas. En lo correspondiente a los encofrados, las actividades zapatas y columnas sobresalen por presentar altos rendimientos. La actividad fundida de elementos en concreto muestra un comportamiento similar para los casos de vigas y columnas; el resultado obtenido para los muros de contención es notablemente bajo y contrasta con el rendimiento total que presenta Construdata [12]. En este proceso no se registraron mediciones para la actividad zapata. En lo que respecta al proceso de desencofrado, no se evidencian grandes diferencias entre los rendimientos de los cuatro tipos de actividades, no obstante sobresalen las columnas y las vigas con rendimientos superiores a una hora por metro cúbico.

En el comparativo que se presenta entre los rendimientos totales y los suministrados por Construdata [12], se evidencia que con excepción de la actividad vigas, todos los rendimientos calculados están por debajo de los de la base de datos siendo el más cercano el correspondiente a la actividad columnas con una diferencia del $10 \%$. La mayor diferencia se presenta con la actividad muros de contención en donde las observaciones arrojaron un valor promedio $70 \%$ menor que el de la referencia. Tan solo en el caso de la actividad vigas el rendimiento observado está $40 \%$ más alto que el de la referencia. En el caso de los rendimientos de la mampostería, las diferencias obtenidas entre el promedio de las observaciones y la base de datos de referencia, son del orden del $50 \%$ por debajo.

\section{AGRADECIMIENTOS}

Los autores expresan sus agradecimientos a los ingenieros Carlos Andrés Gamboa Pico y Eliecer Beltrán Castillo, quienes ejercieron como auxiliares de la investigación en su época de estudiantes de la facultad de ingeniería civil y llevaron a cabo la toma de datos en campo para la elaboración del estudio. De manera especial se agradece a la Universidad Pontificia Bolivariana y a la Dirección General de Investigaciones por el respaldo y apoyo financiero.

\section{REFERENCIAS}

[1] J. G. Consuegra, Presupuestos de Construcción, Bogotá, Bhandar- Legis SA, 2006.
[2] S. Zhigang, W. Jensen, C. Berryman, \& Z. Yimin, “Comparative Study of Activity-Based Construction Labor Productivity in the United States and China", Journal Of Management In Engineering, 27 (2),116-124, 2011.

[3] A. M. Jarkas, "Buildability Factors Influencing MicroLevel Formwork Labour Productivity of Slab Panels in Building Floors", Architectural Engineering And Design Management, 6, 161-174, 2010.

[4] N. Farnad, N. Pouya, "Dynamic modeling of labor productivity in construction projects", International Journal of Project Management, 31 (6), 903-911, 2013.

[5] L. F. Botero, "Análisis de Rendimientos y consumos de mano de obra en actividades de construcción", Revista Universidad EAFIT, 128, 9-22, 2002.

[6] R. F. Navas, M. R. Ridl, L. Torés, "Mano de obra en la construcción: Determinación de la Cuadrilla Optima por medio de una Herramienta de Simulación, Ingeniería", Revista Académica de la Facultad de Ingeniería, Universidad Autónoma de Yucatán, 16 (2), 151-163, 2012.

[7] N. Ovararin, C. M. Popescu, "Field Factors Affecting Masonry Productivity", AACE International Transactions, 9.1, 2001.

[8] L. F. Martínez, R. Verbal, A. Serpell, “Recomendaciones para aumentar la productividad en la construcción", Revista Ingeniería de Construcción, 8, 1-14, 1990.

[9] L. M. Polanco, Análisis de Rendimientos de Mano de Obra para Actividades de Construcción - Estudio de Caso Edificio J UPB, Universidad Pontificia Bolivariana, Bucaramanga, 2009.

[10] G. Mejía, T.C. Hernández, “Seguimiento de la Productividad en Obra: Técnicas de Medición de Rendimientos de Mano de Obra", UIS Ingenierías, 6 (2), 45 - 59, 2007.

[11] A. Cano \& G. Duque, Rendimientos y Consumos de Mano de Obra, Medellín: SENA - CAMACOL, p.43, 2000.

[12] Revista CONSTRUDATA-LEGIS, ISSN: 2322-6552. http://www.construdata.com/ (Última visita febrero de 2014).

[13] A. R. Refaat, E. M. Hany, A. H. Mohamed, "Labor productivity: Benchmarking and variability in Egyptian projects", International Journal of Project Management, 25 (2), 189-197, 2007. 